\title{
Las bondades del software libre en el proceso de enseñanza - aprendizaje en la educación media
}

\author{
Luis Jesús Pedro Criollo ${ }^{1}$
}

Recibido: 17-10-2018

Aceptado: 03-12-2018

\section{Resumen}

El presente artículo tiene como objetivo presentar los resultados de la investigación "la metodología B-learning aprovechando las bondades del software libre en el proceso de enseñanza aprendizaje de la asignatura de informática en el nivel de educación media de la Institución Educativa Municipal Liceo José Félix Jiménez - jornada de la tarde de la ciudad de Pasto". La metodología se inscribió bajo el enfoque mixto de tipo descriptiva, aplicando como técnicas de recolección de la información la observación directa, la revisión documental y la encuesta. La información recolectada permitió establecer cuáles fueron las necesidades y expectativas de formación de la población objeto de estudio, con el fin de que el docente propicie escenarios donde el estudiante se convierta en el protagonista principal y sea él, quien construya sus conocimientos. Asimismo, se da a conocer cuáles fueron las bondades del software libre en el diseño de la metodología B-learning apoyando al alcance de la visión institucional y de algunas directrices del MEN. Se concluye que el software libre permitió crear escenarios de

\footnotetext{
1. Docente medio tiempo de la Facultad de Ciencias y Tecnologías, de la División de Universidad Abierta y a Distancia de la Universidad Santo Tomás, Centro de Atención

Universitaria Pasto. Docente del área de Tecnologia e Informática en la Institución Educativa Municipal Liceo José Félix Jiménez - J.T., adscrita a la Secretaria de Educación Municipal de Pasto.

Pasto - Colombia.

Correo electrónico: jotapece36@hotmail.com - pedrocriollo@ustadistancia.edu.co -

Icriollo16@unab.edu.co

ORCID: http://orcid.org/0000-0002-9723-799X
} 
innovación en los procesos de enseñanza-aprendizaje, al igual que, visualizaron la transversalidad de la herramienta con otras áreas de formación.

Palabras clave: software, software libre, enseñanza, aprendizaje, educación, B-learning, innovación.

\section{The benefits of free software in the teaching - learning process in secondary education}

\section{Abstract}

The objective of this article is to show the results of the research "the B-learning methodology taking advantage of the benefits of free software in the teaching-learning process of the computer science subject at the level of secondary education of the Liceo José Félix Municipal Educational Institution Jiménez - afternoon of the city of Pasto ". The methodology was registered under the mixed approach of descriptive type, applying as techniques of information collection the direct observation, the documentary review and the survey. The information collected allowed us to establish what were the needs and expectations for the training of the population under study, in order that the teacher create scenarios where the student becomes the main protagonist and it is he who builds his knowledge. Likewise, the advantages of free software in the design of the B-learning methodology are explained, supporting the scope of the institutional vision and of some MEN guidelines. It is concluded that free software allowed the creation of scenarios of innovation in the teaching-learning processes, as well as, visualized the transversality of the tool with other training areas.

Keywords: software, free software, teaching, learning, education, B-learning, innovation. 


\section{Introducción}

La época actual despliega innumerables herramientas que permiten el acceso a la información y que, a su vez, generan la necesidad de abrir canales de comunicación, por ser un entorno dinámico y en constante cambio (Aparicio, 2018), herramientas que han logrado generar trasformaciones en la vida del ser humano y por ende en la educación. Los procesos de enseñanza-aprendizaje en el área de informática de la IEM José Félix Jiménez de la Ciudad de San Juan de Pasto, se enmarcan en un proceso tradicional, puesto que, el docente dentro de su didáctica de formación únicamente deja ejercicios, talleres o guías de trabajo impresos, la intensidad horaria es limitada y los grupos de trabajo muy numerosos, esto dificulta el desarrollo del acto pedagógico, desaprovechando los recursos tecnológicos que tiene la Institución y las bondades que ofrece el software libre en los diferentes escenarios.

La investigación tuvo como propósito identificar las necesidades y expectativas de formación, reconocer las bondades que ofrece el software libre, diseñar e implementar la propuesta y valorar su impacto. Las razones que motivaron su realización se enfocan principalmente en la labor del maestro contemporáneo, cuyo reto es fortalecer las competencia y habilidades del educando perfiladas a una sociedad que se relacione con los nuevos entornos de aprendizaje, para este caso el software libre, vista como una posibilidad amplia de herramientas para familiarizarse con la información y la tecnología siendo una premisa fácil y permanente, desafío alcanzable, si se logra trasformar el paradigma tradicional del aprendizaje.

De esta manera, la implementación de la metodología B-learning a través de una plataforma virtual basada en software libre para el área de informática - PEVI, integra diferentes recursos como los foros, chat, cuestionarios, entre otros, permitiendo fortalecer en el educando sus competencias tecnológicas y comunicativas hacia el alcance de un agente activo de su proceso de formación. 
Por lo anterior, el presente artículo da a conocer los referentes teóricos; aspectos metodológicos, los hallazgos más significativos, el impacto de la propuesta en el grupo objeto de estudio y finalmente las conclusiones del mismo.

\section{Acercamientos teóricos - conceptuales.}

Los referentes que sustentaron y orientaron la investigación, parten del proceso de enseñanza - aprendizaje, la importancia del uso de las TIC y las bondades del software libre en la metodología B-Learning, a través de revisión bibliográfica y estudios relacionadas con el tema, con el fin de aportar a la labor docente de la educación media en la implementación de nuevas metodologías que permitan estar a las necesidades del siglo XIX.

Para ello, el aprendizaje requiere de escenarios trasformadores (de la calle, Malaver, Gallego, Rodríguez, Flórez, Saldaña, 2014), de intercambio y complementariedad entre el docente y el estudiante, para ello es necesario propiciar el desarrollo integral del alumno, apoyándose por actividades de enseñanza que permitan la creatividad, que estimulen el aprendizaje y conlleven a transformar el contexto en el que están inmersos.

La enseñanza, por su parte, es «esencialmente un proceso de comunicación, empatía e interacción que promueve la implicación y reciprocidad entre estudiantes, profesorado y otros agentes, que pueden colaborar en la institución educativa» Por su parte, Claxton, G. (2001) plantea que, de algún modo los nuevos enfoques de la educación se están centrando en procesos de aprendizaje en que los profesores están dando valor a la experiencia, el uso de la imaginación, la intuición, la colaboración, y en actitudes de compromiso con problemas e incertidumbres de la vida real, reforzando así, la capacidad de los alumnos y la disposición a asumir la responsabilidad de su propio aprendizaje. Partiendo de esta definición, es importante enseñar a los estudiantes a ser estratégicos y recursivos, para que puedan alcanzar sus objetivos, teniendo en cuenta el entorno que los rodea. 
Por lo tanto, las TIC en el proceso educativo pueden ayudar a generar y dinamizar los procesos de enseñanza-aprendizaje para despertar, sostener y proyectar el interés por el aprendizaje, buscando que este cobre sentido para el estudiante y promueva el enlace entre los saberes y la trascendencia para el desarrollo personal (Aparicio \& Ostos, 2018).

Por otra parte, la institución como un todo, ofrece al estudiante una actividad orientada pedagógicamente, se puede integrar con el plan estratégico de la organización, generando un sinnúmero de ventajas, entre ellas, mejora la utilización del aula de clase, puede ayudar a ser más congruente el servicio prestado con las necesidades de los estudiantes (Ferreyra, 2014) y puede ayudar a cumplir con los cursos y programas ya en marcha. Para el docente, les permite el acceso a nuevos recursos, los introduce en el modelo de la educación virtual, es una oportunidad para el desarrollo profesoral y para experimentar con nuevas técnicas pedagógicas, ayuda a conocer las expectativas de los estudiantes y a promover el desarrollo de sus habilidades, permite una programación más flexible en el tiempo y mantiene la parte de la presencialidad que los profesores pueden valorar más.

Estas ventajas, permiten una integralidad entre los actores del proceso formativo, incluso favorecen la planificación y el desarrollo de diseños y planes curriculares, con base a los requerimientos legales y de contexto, a fin de responder a la evolución, trasformación y exigencia de la sociedad (Ortiz, Buitrago, 2017). Los alumnos trabajan en grupo, aplicando el conocimiento combinado con el fin de solucionar el problema.

Lo anterior permite señalar que el B-Learning es una estrategia innovadora, dado que integra las TIC a las prácticas pedagógicas, mejora la interacción entre todos los integrantes de la comunidad educativa, aumenta la motivación y los procesos de enseñanza se centran en el estudiante.

Ahora bien, a fin de entender a groso modo las bondades del software libre, Gonzalez, J. C. (2007) señala que el software libre se 
refiriere "a la libertad de los usuarios para ejecutar, copiar, distribuir, estudiar, cambiar y mejorar el software". Además, éste aplicado a la educación "favorece el proceso de enseñanza-aprendizaje en función de los destinatarios, de sus necesidades, su nivel de formación ya que puede ser modificado y adaptado en función de nuestros intereses y de los objetivos que persigamos".

Por lo anterior, hablar de software libre se refiriere a ese espacio de libertad que permite a los usuarios ejecutar, copiar, distribuir, estudiar, cambiar y mejorar el software, según necesidades particulares atendiendo a una finalidad y principios particulares y misionales, generando la libertad para mejorar el programa y luego publicarlo para el bien de toda una comunidad (Viejo, Cabezas, Martínez, 2013) -el acceso al código fuente es condición indispensable para esto-.

En este orden de ideas, es importante, además, reconocer la principal funcionalidad de un entorno virtual de aprendizaje el cual entre muchas de sus bondades permite ser un repositorio de información útil de aprendizaje, un lugar para poner a disposición de los estudiantes todo tipo de documentos y también sitios web, blogs con enlaces a otros sitios de contenido (Konieczny, 2015). De hecho, se trata de romper esas barreras de espacio/tiempo.

También hay que tener en cuenta que, un entorno virtual de aprendizaje debe de ser accesible desde cualquier sitio $y$, en estos momentos, también desde cualquier dispositivo. Por último, se puede decir que los EVAS no están delimitados a la formación online o a distancia, sino que son una herramienta complementaria a la formación presencial en muchas ocasiones.

Las anteriores características, permite establecer que, dentro del proceso educativo siempre estará presente un entorno de aprendizaje. En ese sentido, los Entornos Virtuales de Aprendizaje son espacios creados con tecnología electrónica en los que tienen lugar procesos de aprendizaje.

Por consiguiente, es el espacio virtual en el que han de coincidir los participantes, entonces, un ambiente virtual de aprendizaje se 
configura como un sistema de software que se diseña para facilitar la gestión de cursos virtuales a través de los cuales se establece una relación entre el docente y los estudiantes en el marco del proceso educativo. Estos entornos virtuales permiten la administración, desarrollo y control de los cursos de tal manera que se pueda hacer un seguimiento detallado del aprendizaje de los estudiantes. Generalmente son diseñados para el desarrollo de cursos virtuales, sin embargo, son bastante útiles para apoyar y complementar los procesos educativos presenciales combinando la presencialidad y la virtualidad.

Finalmente, para el ejercicio investigativo, fue importante la revisión de antecedentes respecto al tema, dado que, con base a estos, los aportes y conclusiones, permite enriquecer la estructuración del recorrido a seguir frente a la identificación de referencias que soporten el proceso metodológico. Al respeto, se encuentran los siguientes:

- Indagar sobre software libre y B-learning en el campo educativo como una metodología novedosa que permite el aprovechamiento de las TIC en los procesos de enseñanza-aprendizaje en el área de informática, conlleva repensar la implementación de estrategias, materiales didácticos, procesos evaluativos que favorezcan la calidad de las instituciones.

- La implantación del modelo B-Learning en la Educación resulta muy factible de realizarse, por el hecho de tratarse de una plataforma gratuita, fácil de usarse y con requerimientos técnicos mínimos, además, el debate enlínea favorece la emergencia de un discurso progresivo al facilitar la interrelación e integración de ideas sobre las diferentes situaciones. [2] Los podcasts promueven en los estudiantes la habilidad en la producción escrita, la autonomía de aprendizaje, y aumenta su motivación por aprender idiomas. 
- La implementación de un b-learning, como alternativa pertinente en las instituciones de educación que deseen enfrentar los nuevos desafíos se convierte en un proceso con dimensiones amplias que necesita de una unidad central responsable de conducir, facilitar y habilitar su desarrollo, de acuerdo a los intereses, lineamientos y cultura de la institución. Por su parte, Meza-Jaque, J. \& Badillo, G. (2009concluyen que, este modelo permite mejorar la actividad académica de los diferentes cursos impartidos por la Escuela además de establecer vínculos formales con los alumnos y profesores de los cursos, mejorar la percepción de pertenencia de los alumnos y profesores.

- Lara-Jimenez (2010) efectuó la investigación «Apropiación de la plataforma Virtual SAKAI y análisis de funcionalidad con la plataforma MOODLE», cuyo objetivo fue realizar un análisis de funcionamiento de los componentes de las plataformas tomando en consideración aspectos como los de instalación y de implementación, como también la operatividad que ofrecen estas herramientas. Este estudio aporta a la propuesta puesto que en sus anexos se encuentran los manuales de usuario de la plataforma Moodle que fueron útil para la implementación de la metodología b-learning con un aula virtual basada en software libre.

\section{Aspectos metodológicos.}

A fin de dar cumplimiento al alcance de los objetivos de investigación desde las bondades del software libre, en el proceso de enseñanza aprendizaje en la educación media, el tipo de investigación al cual se suscribe este estudio es Investigación Descriptiva dado que, se llega a conocer la situación y actitudes predominantes a través de la descripción exacta de las actividades, objetos, procesos y personas. Para Hernández-Sampieri, R. (2014) la ruta metodológica seguida se inscribe en el enfoque mixto, el cual permite 
al investigador "producir datos más "ricos" y variados mediante la multiplicidad de observaciones, ya que se consideran diversas fuentes y tipos de datos, contextos o ambientes y análisis". [8] La muestra está conformada por el docente y 90 estudiantes matriculados en la educación media de los grados 10 y 11 a la asignatura de tecnología e informática.

Se emplearon diversas técnicas de recolección de la información, a nivel cualitativo se encuentran la observación directa que permitió recoger información de manera participativa necesaria para identificar los elementos de la metodología B- learning en los procesos de enseñanza-aprendizaje en el área de Tecnología e Informática; revisión documental, específicamente de carácter institucional dado que se convirtieron en un parámetro para la construcción de la plataforma en cuanto al trabajo colaborativo y la forma de evaluación, la exploración de tutoriales, manuales, videos, entre otros relacionados con la implantación de la metodología B- learning y plataformas virtuales basados en software libre. Desde la parte cuantitativa se aplicó una encuesta estructurada online.

Después de la recolección de la información se procedió al análisis de la misma, permitiendo al investigador triangular la realidad a través de documentos de diferente materialidad, con el fin de acreditar las justificaciones e interpretaciones y reconstrucción del fenómeno que tiene características de historicidad.

\section{Resultados de investigación.}

Con la recolección de información se dio paso a la sistematización, análisis e identificación de hallazgos, generando los siguientes resultados.

- Algunos estudiantes carecen de comprensión o asimilación del concepto y la relevancia que tienen las TIC dentro de los diferentes escenarios a los que se enfrentan en 
su cotidianidad, dado que únicamente la toman como un instrumento para ingresar a las diferentes redes sociales, descargar videos, música, juegos, entre otros, poco provechosos para su formación integral.

- Las estrategias actuales utilizadas dentro del proceso de formación-aprendizaje en el área de aprendizaje, no genera espacios que favorezcan al estudiante adaptarse a su propio estilo y ritmo de aprendizaje, especialmente con los estudiantes con necesidades educativas especiales.

- El tiempo destinado para las clases de informática es limitado, ya en el momento cuentan con una intensidad horaria semanal de 2 horas, por lo tanto, se requiere este tiempo y además el tiempo libre con el que cuentan los estudiantes.

- Los exalumnos se ven enfrentados a plataformas educativas virtuales, lo que en principio es difícil adaptarse a ellas, por lo tanto, se requiere que los estudiantes de la media se capaciten en el manejo de estas plataformas para poder afrontar las exigencias de la educación superior y la vida laboral.

- La visión institucional establece que la Institución educativa será reconocido a nivel regional por su innovación pedagógica, incluso refiere que habrá conocimiento y aplicación de nuevas herramientas informáticas, dentro de los procesos de formación.

- Se evidencia en la clase de informática que los estudiantes se sienten motivados por aprender el funcionamiento de nuevos programas informáticos que les permita enfrentarse a los retos que la sociedad les exige, al igual que, comprender los nuevos conceptos que el área de informática les presenta. 
- Dentro del contexto educativo se plantea diferentes desafíos, los cuales deben responder a las necesidades y expectativas específicas de cada comunidad, es así que, en la población objeto de estudio se identificaron diversas necesidades y expectativa que sirvieron de base para la construcción de la metodología B-learning basada en software libre, entre ellas se encuentra que, en el desarrollo de la clase de informática los estudiantes del grado $10^{\circ}$ y $11^{\circ}$ en su mayoría esperan recibir instrucciones precisas por parte del docente, les falta compromiso por la clase, se les dificulta tomar decisiones para la resolución de situaciones problemas, administrar, regular y evaluar su forma de aprender.

Los anteriores hallazgos permitieron establecer que, la población objeto de estudio requiere fortalecer el aprendizaje autónomo, entendido éste como la capacidad que debe poseer el educando para regular sus propias actuaciones sin depender que otros lo hagan, logrando que tome sus propias decisiones, asumiendo los efectos y buscando el beneficio frente al proceso de enseñanzaaprendizaje. Ésta situación establece la importancia de trabajar en el desarrollo de competencias para el manejo de las herramientas tecnológicas y digitales y la responsabilidad en el tratamiento de la información, aspectos éstos que conlleven abordar el área de informática como: "herramienta que permite desarrollar proyectos y actividades tales como la búsqueda, la selección, la organización, el almacenamiento, la recuperación y la visualización de información", al igual que, el reconocimiento de su uso es, "un derecho que implica deberes en su conducta por respeto consigo mismo y con los demás" Colegio de la Salle (2010), consideraciones estas que fueron tenidas en cuenta en la implementación de la metodología B-learning basada en software libre en la institución educativa, convirtiéndola en una herramienta útil para que el docente ponga en marcha el diseño y planificación de escenarios educativos que permitan el desarrollo de dichas competencias.

Otro aspecto encontrado en la observación, es la falta de trabajo en equipo, dado que se les dificulta asumir roles conjuntos 
para alcanzar el desarrollo eficiente de actividades o tareas que involucren poner en marchas sus diferentes actitudes y aptitudes frente a temáticas y situaciones problémicas que conlleven a fin común. Esto permite al alumno adaptarse a su propio estilo y ritmo de aprendizaje; alcances que asume la metodología B-learning, reconociendo las bondades que ofrece el software libre por su amplitud de escenarios y herramientas que motivan al despliegue de didácticas que favorecen la profundización del conocimiento, según habilidades cognitivas y de comunicación.

Una última necesidad observada se centra en la innovación didáctica, dado que algunos estudiantes demuestran haber experimentado un proceso de aprendizaje tradicional donde la formación en tecnología e informática fue impartida en su mayoría con prácticas pedagógicas tradicionales donde el trabajo se centraba en desarrollar actividades académicas con base a los lineamientos del MEN en espacios solo dentro del aula de informática, sin adentrarse en otros escenarios de la era digital. Razón por la cual, el docente debe propiciar escenarios, utilizando asertivamente las TIC y el estudiante se convierte en el protagonista principal, dado que la información y comunicación permiten que él contribuya a la construcción de los conocimientos y aprendizajes.

\section{Plataforma educativa virtual informática - PEVI}

Con base a los hallazgos identificados se diseñó e implemento la metodología B-learning apoyada en la PLATAFORMA EDUCATIVA VIRTUAL INFORMATICA (PEVI) basada en software libre para los procesos de enseñanza aprendizaje en la asignatura de informática en el nivel de educación media, donde se tuvieron en cuenta las siguientes fases y consideraciones, según aportes de Herrera-Batista, M.A. (2006):

1) Diseño: Dentro de las bondades del software libre se encuentra la posibilidad de crear ambientes virtuales de aprendizaje apoyados en una plataforma virtual desarrollada Moodle, que, entre otras ventajas, permite contemplar componentes presenciales y virtuales. 


\section{DISEÑO}

\section{METODOLOGIA B-LEARNING}

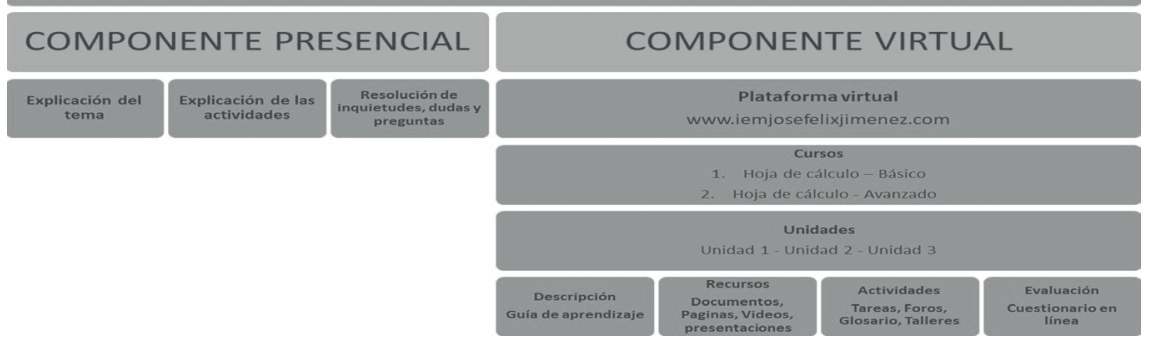

Figura 1. Diseño de metodología b-learning.

2) Implementación: Para la creación de la plataforma PEVI fue necesario la consecución a los servicios de la empresa WinkHosting en dominio llamado http://www.iemjosefelixjimenez.com y hosting con una capacidad de 350 megabytes de almacenamiento. Así mismo, se contemplaron los re Requisitos para la plataforma Moodle, dado que está desarrollado principalmente en GNU/Linux usando Apache, PostgreSQL, MySQL, MariaDB y PHP (también conocida como plataforma LAMP). Esta es la combinación más segura y más común. Otros aspectos importantes dentro del proceso de implementación fue la creación del aula virtual, creación de cursos, añadir usuarios, presentación de la plataforma y la explicación del docente.

\section{Impacto plataforma PEVI}

Para valorar el impacto del uso de la metodología B-learning usando software libre en los procesos de enseñanza-aprendizaje de la asignatura de informática, se aplicó a noventa estudiantes de los grados 10 y 11, una encuesta on line, con nueve preguntas de selección múltiple, la cual arroja los siguientes resultados e interpretaciones.

EL 53,3\% de la población encuestada, utilizó entre 2 y 4 horas y un $30 \%$ más de 4 horas, la plataforma PEVI. Esta información per- 
mite analizar que, el $83 \%$ de población está motivada en el manejo asincrónico de la plataforma, dado que, les facilita que trabajen desde cualquier lugar, con las mismas herramientas que utilizan en la institución de forma legal y gratuita, favoreciendo la continuidad en su proceso de aprendizaje. Por consiguiente, la metodología B-learning, basada en software libre, favorece también el aprovechamiento del tiempo libre, donde los estudiantes pueden desarrollar de manera autónoma diferentes actividades, entre ellas las académicas, que contribuye a mejorar su rendimiento académico.

Además, beneficia a la Institución Educativa, en el mejoramiento de la calidad de la educación, dado que, da seguimiento a la funcionalidad del proceso de formación de los educandos, a la interacción entre sus actores, haciendo que la enseñanza y el aprendizaje sean innovadores.

En cuanto a la calificación de la plataforma, el 72,2 \% calificó la plataforma PEVI en un nivel excelente y un $27.8 \%$ en un nivel bueno, esto permite inferir que la población objeto de estudio está satisfecha con la plataforma como un nuevo medio que apoya los procesos de aprendizaje, puesto que, ésta metodología basada en software libre se convierte en apoyo a las actividades escolares, como una herramienta que permite fortalecer el pensamiento a través de la elaboración de sistemas de ofimática, creación de mapas mentales, creación de diagramas de flujo, manipulación de imágenes, etc. También fortalece las competencias comunicativas mediante la utilización del correo electrónico, foros de discusiones, chat, entre otros. Otro aspecto a destacar en esta plataforma es el acceso a recursos de información en línea.

En la identificación de algunas desventajas, la población objeto de estudio, estableció que puede existir alguna dificultad en el uso de la plataforma en cuanto, a la baja o nula conectividad, situación que se sale de las manos, dado que es una condición externa. Sin embargo, si se puede, crear espacios de reflexión con los estudiantes para la adecuada estructuración metodológica y organización de cronogramas o agendas de trabajo. 
Por lo anterior, se puede afirmar que, el aprendizaje del educando no solo está relacionado a la interacción del estudiante con los saberes, contenidos y actividades a través del uso de las TIC o sus herramientas, sino que además requiere de una metodología que orientada desde acciones de asesoría y acompañamiento, motiven y ayuden al estudiante en la adquisición de valores y condiciones desde la autonomía; interacción requeridas para fortalecer la competencia socio-afectiva de respeto y dialéctica que siempre deberá existe entre maestro y estudiante, ejerciendo una regulación recíproca entre los participantes, de ahí la importancia de una adecuada metodología .

En lo referente al nivel de satisfacción los estudiantes afirmaron que, el $70 \%$ se encuentran completamente satisfechos, seguido del $30 \%$, quienes se encuentran bastante satisfechos, aspectos que permiten establecer que la plataforma PEVI basada en software libre, contribuye a que los educandos conozcan cómo funcionan los programas y fomenta el interés por la adquisición de nuevos aprendizajes y conceptos en el campo de la informática, los cuales son relevantes y útiles para su contexto a mediano y largo plazo.

Haciendo la comparación entre la metodología anterior y con la propuesta, el 75,6\% se encuentra completamente satisfecho con la metodología mediada por la plataforma PEVI porque, es una herramienta que en primera instancia contribuye al fomento adecuado de la comunicación entre maestro y estudiante, facilita el acceso a la información con la variedad de actividades individuales y colaborativas, desarrollando habilidades y competencias requeridas en la actual sociedad de la información.

\section{Conclusiones}

El trabajo expuesto es el resultado de un proceso investigativo llevado a cabo con la intensión de implementar la metodología Blearning aprovechando las bondades del software libre en el proceso de enseñanza - aprendizaje de la asignatura de informática 
en la media, convirtiéndose en una herramienta que permitió el desarrollo de la motivación de los estudiantes, puesto que les facilitó el acceso al conocimiento, el trabajo colaborativo y autónomo $y$, sobre todo desarrollar competencias para la resolución de problemas, llevando a la institución educativa a generar innovación pedagógica y ser un referente para las instituciones educativas de la ciudad.

Razón por la cual, el docente debe propiciar escenarios donde el estudiante se convierta en el protagonista principal y sea él, quien construya sus conocimientos (Aparicio \& Ostos, 2018). También, al diseñar e implementar la metodología B-learning utilizando el software libre en los procesos de enseñanza - aprendizaje permitió crear escenarios de innovación y transformación de paradigmas tradicionales dentro de las practicas pedagógicas, haciendo que los aprendizajes sean más significativos en miras de la construcción del proyecto de vida del educando.

Además, el valorar el impacto del uso de la metodología B-learning usando software libre en la asignatura se obtuvo un significativo porcentaje de apreciaciones en un nivel de satisfacción alto al ser evaluada por los estudiantes como una nueva herramienta que apoya el proceso de aprendizaje y el proyecto de vida.

\section{Referencias bibliográficas}

Aparicio, O.Y. (2018). Las TIC como herramientas cognitivas. Revista Interamericana de Investigación, Educación y Pedagogía, RIIEP, 11(1). https://orcid.org/0000-0003-3535-6288

Aparicio, O.Y., \& Ostos, O.L. (2018). Las TIC como herramientas cognitivas para la investigación. Revista Interamericana de Investigación, Educación y Pedagogía, RIIEP, 11(1). https://orcid.org/0000-0003-3535-6288. https://orcid.org/0000-0002-6477-9872

Aparicio, O.Y., \& Ostos, O.L. (2018). El constructivismo y el construccionismo. Revista Interamericana de Investigación, Educación 
y Pedagogía, RIIEP, 11(2). https://orcid.org/0000-0003-3535-6288. https://orcid.org/0000-0002-6477-9872

de la Calle, C. V., Malaver, M. O., Gallego, J. D. M., Rodríguez, M., Flórez, J. C., Henao, C. E. \& Saldaña, R. (2014). Aportes de los doctorados de educación en ciencia, tecnología y sociedad, desde la sistematización de sus investigaciones doctorales científicas y formativas, 2000-2010. Revista Interamericana de Investigación, Educación y Pedagogía, RIIEP, 7(1). DOI: https://doi.org/10.15332/s1657-107X.2014.0001.04

Fernández, C., Llinares, S. \& Valls, J. (2011). Características del desarrollo de una mirada profesional en estudiantes para profesor de matemáticas en un contexto b-learning. Acta Scientiae, 13(1), 9-29.

Ferreyra, H. A. (2014). Mesas Socioeducativas para la Inclusión y la Igualdad. Un programa "De todos con todos". Una experiencia en construcción. Revista Interamericana de Investigación, Educación y Pedagogía, RIIEP, 7(2). DOI: https://doi.org/10.15332/s1657107X.2014.0002.01

González-Mariño, J. C. (2006). B-Learning utilizando software libre, una alternativa viable en Educación Superior. Revista Complutense de Educación, 17(1), 121-133.

Meza-Jaque, J. \& Badillo, G. (2009). Modelo B-Learning en la Escuela de Informática - UNAB - Chile. Virtual Educa. Congreso. Buenos Aires Argentina.

Lara-Jimenez, E.A. (2010). Apropiación de la plataforma Virtual SAKAI y análisis de funcionalidad con la plataforma MOODLE (tesis de pregrado), Universidad de Nariño, Pasto - Nariño. Recuperado de udenar.edu.co.

Claxton, G. (2001). Aprender. El reto del aprendizaje continuo. Barcelona: Paidos.

Colegio de la Salle (2010). Tecnología e informática. Recuperdo de http://www.sallemonteria.edu.co/index.php/areas-academicas-lasallemonteria/informatica

Gonzalez, J. C. (2007). B-Learning utilizando software libre,. CienciaUAT, 60-66. 
Hernández Sampieri, R. et al. (2014). Metodología de la investigación. México D.F.: Mcgraw-Hill/Interamerican Edictores, S.A. DE C.V. p.537

Herrera-Batista, M.A. (2006). Consideraciones para el diseño didáctico de ambientes virtuales de aprendizaje: una propuesta basada en las funciones cognitivas del aprendizaje. Revista Iberoamericana de Educación, pp.120. Recuperado de file:///D:/Downloads/1326Herrera\%20(1).pdf

Konieczny, P. (2015). Lorenzo García Aretio: bases, mediaciones y futuro de la educación a distancia en la sociedad digital. Revista Interamericana de Investigación, Educación y Pedagogía, RIIEP, 8(1). DOI: https://doi. org/10.15332/s1657-107X.2015.0001.08

Ortiz, J. G., \& Buitrago, H. (2017). La evaluación en la tradición educativa colombiana. Instrumento de clasificación social. Revista Interamericana de Investigación, Educación y Pedagogía, RIIEP, 10(1), 145-171. DOI: https://doi.org/10.15332/s1657-107X.2148

Viejo, C. M., Cabezas, I. L., \& Martínez, M. D. J. I. (2013). Las redes de académicas en la docencia universitaria. Revista Interamericana de Investigación, Educación y Pedagogía, RIIEP, 6(2). DOI: https://doi. org/10.15332/s1657-107X.2013.0002.03 